\title{
Graft-versus-T-Cell Non-Hodgkin's Lymphoma Effect
}

\author{
Liang-Tsai Hsiao, Tzeon-Jye Chiou, Wei-Shu Wang, Po-Min Chen \\ Division of Hematology \& Oncology, Department of Medicine, Taipei Veterans General Hospital; \\ National Yang-Ming University School of Medicine, Taipei, Taiwan
}

Received November 28, 2005; received in revised form December 27, 2005; accepted January 6, 2006

Int J Hematol. 2006;83:193. doi: 10.1532/IJH97.05176

(C)2006 The Japanese Society of Hematology

A 48-year-old man received a diagnosis of peripheral T-cell lymphoma, unspecified, of Ann Arbor stage IV, with multiple skin lesions on the chest and left forearm. He experienced a complete remission after chemotherapy. Later the patient received an HLA-matched allogeneic bone marrow transplantation; however, the skin lesions rapidly recurred 3 months posttransplantation. The findings of a skin lesion biopsy revealed infiltrations of neoplastic $\mathrm{CD}^{+} \mathrm{T}$-cells in the epidermis and upper dermis (Figure 1, Hematoxylin-Eosin staining). Donor lymphocyte infusion (DLI) was given to reverse the relapse. Unfortunately, fever and generalized skin rash developed 1 week later, and acute graft-versus-host

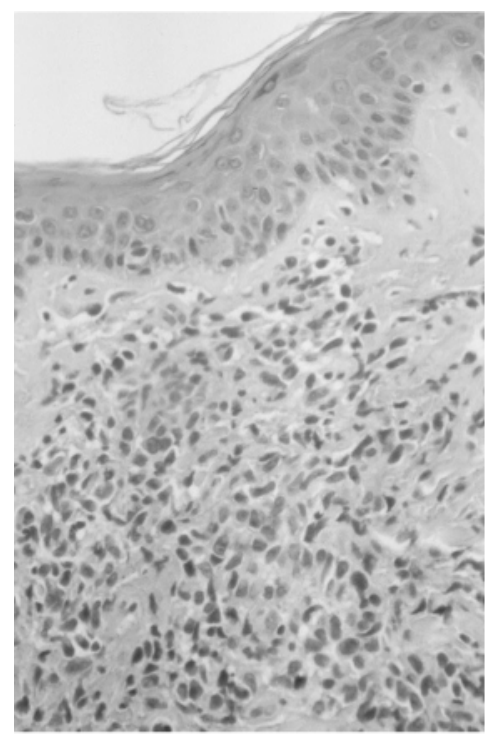

Figure 1. disease (GVHD) of grade IV was diagnosed. The biopsy of previous skin lesions performed at the third week after DLI showed a nearly complete disappearance of lymphoma cells (Figure 2, Hematoxylin-Eosin staining). The patient died 4 weeks after DLI because of acute GVHD.

Peripheral T-cell lymphoma is frequently diagnosed at an advanced stage, manifesting with $\mathrm{B}$ symptoms and skin and marrow involvement, and has a poor prognosis by a relatively low response rate and a high relapse rate. Long-term remission has been reported in several cases after allogeneic transplantation, with the graft-versus-T-cell non-Hodgkin's lymphoma effect implied.

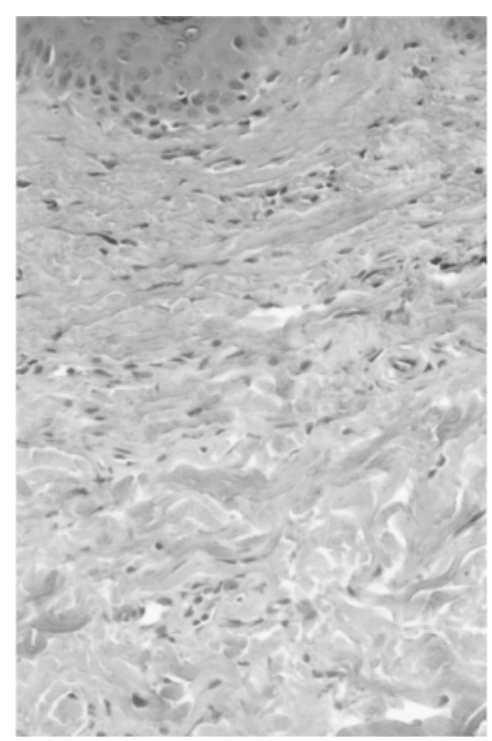

Figure 2.

Correspondence and reprint requests: Liang-Tsai Hsiao, MD, Division of Hematology \& Oncology, Taipei Veterans General Hospital, No. 201, Sec. 2, Shih-Pai Road, Taipei 11217, Taiwan; 886-228712121 \#2507; fax: 886-2-28732184 (e-mail: lthsiao@vghtpe.gov.tw). 